\title{
Data Analysis Application to Investigate Relationships between Metacognition and Learning Styles
}

\author{
Anass Hsissi, Hakim Allali, Abdelmajid Hajami \\ LAVETE - e-learning Research Lab, University of science \& technology, University Hassan I, SETTAT, \\ Morocco \\ anasshsissi@gmail.com
}

\begin{abstract}
The purpose of this study is to examine if learning styles predict effectiveness in learning. Participants of the study consisted of 80 students selected from different classes of the university of science and Technology -Settat, Morocco, two questionnaires on learning styles and metacognition related to reading and comprehension were handed out to members of the sample during academic year 2016/2017. Data analysis techniques were used to understand our dataset; principal component analysis was used to discover significant patterns within data. Results indicate that there are significant relations between the dimensions of learning styles, metacognition and performance in comprehension activity. Results also indicate that learning styles significantly explain and predict all sub-dimensions of metacognition.

As perspective for our research, we tend to offer to each student the appropriate environment and conditions to enhance learning, in the conclusion we will discuss the implications and recommendations for designing an adaptive learning and intelligent environment based on an identification of students' profiles.
\end{abstract}

Keywords: component; learning styles, metacognition, data analysis, PCA, learning environment

\section{Introduction}

The term metacognition was first brought to the literature by J.H Flavell in his research on meta memory functioning [1], it is defined in a large quantities of research papers, as thinking about thinking, or cognition about cognition. It takes place on every learning process, it allows students to control and monitor their own learning, such as selecting the appropriate strategies, intervening, monitoring the execution and even evaluating its effectiveness. Metacognition is a conscious processes, according to many authors [2], [3],[4] metacognition is described as the second order level of cognition.

According to [5], metacognition is a state of awareness and knowledge of one's mental processes such that one can monitor, regulate and direct them to a desired goal.

Livingstone [6] suggests that metacognition refers to higher order thinking, that involves active control over the cognition process which influences the learning success significantly, because students with high metacognitive and self-regulatory abilities are more successful. Metacognitive skills involve the process of individuals deciding what strategy to use in what situations as a result of the metacognitive experiences 
they have gone through, using a strategy, monitoring learning, changing that strategy if learning has not taken place and trying a new one.

We summarize all the ideas, and confirm that metacognition is an important element of cognition, because it's about students knowing and progressing, it helps students retain more information and guides them on how to proceed to be more successful to increase achievement [7].

This positive role of metacognition in learning - and comprehension activity is supported by three theories of learning; cognitive development, behavioristic and information processing learning theories [8]. The role is illustrated by a series of papers and experimental studies to assess the impact of metacognitive skills on learning performance across many disciplines; therefore, our objective is, firstly, to examine the relationship between metacognition and learning styles among university students. Secondly, we tend to classify the sample of students according to their learning styles and metacognitive abilities in reading and comprehension activities.

In the next part of the paper, we expose metacognition more deeply, we will present the nature of reading for comprehension, we will see what is the characteristics of good reader, and we will highlights relations between learning styles and control and awareness.

\section{Metacognition while reading - comprehension activities}

Students use a variety of strategies to work with texts, they take actions to deal with information in the text, and to manage and monitor their understanding intentionally and carefully. While reading, students sometimes need to apply some problem solving strategies to clear up misunderstandings that could arise; on the other hand, some strategies are used as basic mechanisms to aid reading comprehension, for example: note taking, underlining and highlighting textual information, summarizing, etc...Therefore, reading is more than decoding print, it is not just a basic skill, and it is a complex process that refers to a particular world of knowledge and experience.

We are going to underline the relationships between reading for understanding and metacognition defined earlier; we present our conception of the nature of reading as a brief outline of what we have learned from recent research and observations.

\subsection{Nature of reading for comprehension}

Reading is a process; the major product consists of creating a mental representation of the text, which serves as an evolving reference for understanding subsequent parts of the text. As the students read further, they test every meaning and start to monitor their understanding; at every moment they can draw a variety of strategies to readjust their progress, it is about taking distance between oneself and the text content.

Reading is not only words and sentences on the paper, but also the ideas, memories, and personal knowledge evoked by those words and sentences, furthermore, a person who understands one genre of a text is not necessarily proficient at all genres, a chemistry teacher may feel completely insecure when trying to understand some of the original source history materials, while a political science undergraduate can understand more and better. In other words, reading is a complex process, influenced by multiple and situational factors, and needs to be controlled and managed $s$ 
Anass Hsissi, Hakim Allali, Abdelmajid Hajami; Data Analysis Application to Investigate Relationships between Metacognition and Learning Styles, Transactions on Machine Learning and Artificial Intelligence, Vol 5 No 4, August, (2017); pp: 8-19

\subsection{Characteristics of proficient readers}

Different research papers about reading describes some characteristics of good or proficient readers, they possess a set of key habits which could be summarized as follows according to [9].

- Motivated to read and to learn,

- Mentally engaged,

- Socially active around reading tasks,

- $\quad$ Strategic in monitoring the interactive processes that assist comprehension:

$\checkmark$ Setting a goal that shapes their reading processes,

$\checkmark$ Monitoring their emerging understanding of a text, and

$\checkmark$ Coordinating a variety of comprehension strategies to control the reading process.

To that end, the awareness level of the students can be predicted by the use of metacognitive reading strategies, which can inform us about the reading proficiencies, according to [10].

Novice reader's score lower than good readers in using all reading strategies, especially some sophisticated cognitive and metacognitive strategies, because they (novice readers) seem often oblivious to these strategies and the need to use them.

\subsection{Metacognitive strategies in reading for comprehension}

Metacognitive strategies are defined by [11] as: Behaviors undertaken by the learners to plan, arrange, and evaluate their own learning, such strategies include directed attention and self-evaluation, organization, setting goals and objectives, seeking practice opportunities, and so forth.

In the context of reading, self-monitoring and correction of errors are further examples of metacognitive strategies.

A classification of reading strategies is presented by Oxford [12], in line with [11] and [13], they are defined as:

- $\quad$ Cognitive reading strategies used to manipulate the language that includes note taking, summarizing, paraphrasing, predicting, analyzing and using context clues.

- Memory reading strategies, which are techniques used to assist the learner to recall information, such as word association and semantic mapping.

- $\quad$ Compensation reading strategies, such as 'inferencing', and guessing while reading, which can assist the learner in making up for reading deficiencies.

- $\quad$ Affective reading strategies, which include self encouraging behavior to lower anxiety, such as rewarding oneself for reading efficiently.

- Social reading strategies, involving collaborating with peers, for example, to ask questions, seek help or correction and to get feedback while reading.

It's evident that the use of these strategies deals with an additional factor of learning; because every student is different, every student has his own way in which he approaches and responds to a learning experience according to his own learning style. In general, metacognition is the engine that drives self directed learning [14]. 


\section{Learning styles and metacognition}

Learning styles refers to different approaches or ways of learning, students take in information in different ways, by seeing and hearing, reflecting, and acting, reasoning logically and intuitively analyzing and visualizing, therefore our teaching methods also vary from one another. When mismatches exist between learning styles and the teaching manner used in class, or the pedagogy employed in the learning environment, the students may become bored, inattentive, get discouraged, and in some cases abandon the task of learning altogether.

To overcome these problems, a differentiation should take place in a way that all students learn in a manner they prefer according to their style of learning, this can increase comfort and willingness to progress and learn. Recent research claims that learning styles can hurt learning; we will discover those results in discussion section to show you how it is suggested to deal with learning styles.

\subsection{Assessment tools of learning styles}

Using a variety of assessment tools, individuals can discover their own interest levels for a set of criteria to help establish the methods in which they could obtain much information and improve their learning, one assessment tool that can be used in establishing a person's learning style is the index of learning styles (ILS), the ILS result provides an indication of learning preferences, possible strengths and tendencies or habits that might lead to difficulty. The instrument used to assess preferences on four dimensions (active/reflective, sensing/intuitive, visual/verbal, and sequential/global) of the learning style model developed by Richard M. Felder and Barbara A. Soloman of North Carolina State University [15].

Table 1. Description of learning style scales

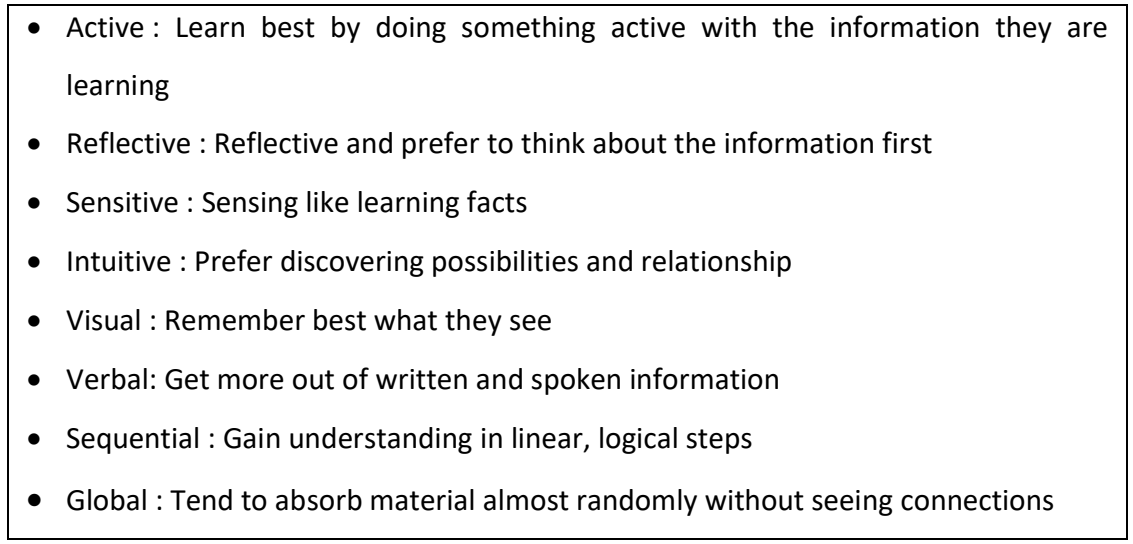

\subsection{Relationship between metacognition and learning styles}

One of the main difficulties that student faces while trying to develop their understanding is an overall lack of awareness to their own learning process [1]. Flavell describes three basic types of awareness related to metacognitive knowledge, the first one is an awareness of knowledge, which is described as an understanding of what the student does and does not know, and what he wants to know, the second one is an awareness of thinking, which describes her understanding of cognitive tasks and the nature of what is required to complete them successfully, finally, there is an awareness of thinking strategies, defined as an understanding of approaches and strategies to regulate learning progress.

Focusing on the function of metacognition itself, there is agreement upon its operational definition. It is a manifestation of the monitoring and regulatory function. 
Anass Hsissi, Hakim Allali, Abdelmajid Hajami; Data Analysis Application to Investigate Relationships between Metacognition and Learning Styles, Transactions on Machine Learning and Artificial Intelligence, Vol 5 No 4, August, (2017); pp: 8-19

Efklides[16] presented three facets of metacognition distinguished by their manifestations as a function of monitoring and control.

Monitoring functions are metacognitive knowledge, knowledge about one's cognition, metacognitive experiences, and metacognitive judgment and assessment which have a very significant impact on learning experience, the control function of metacognition is metacognitive skills; it's about the knowledge of the procedures needed to control cognition. However, the existing literature has paid less attention to the sources of metacognition; the starting points of the construction of metacognition. As mentioned above, individual differences play a critical role because students learn differently and possess differing conceptual systems according to their learning styles and approaches.

\section{Significance of the study}

The basic goal of this study is to determine the relationships between metacognition and learning styles of the students attending the University of Science and Technology. Additionally, the final objective of our study on metacognition is to find how an ideal learning environment could support metacognition in learning as an important dimension of effective learning.

\subsection{Materials and method}

- $\quad$ Population and sample of the study

A total of 80 University students were involved in this study, data were collected in the first hand to measure the student's metacognition abilities in reading and comprehension as well as to determine their learning styles according to ILS model.

- $\quad$ Instruments

Two survey instruments were used in this study, each is described as follows:

\subsubsection{Survey of reading strategies}

The Survey of Reading Strategies Questionnaire (SORS) was developed by Mokhtari and Sheorey (2002)[17] and used to collect data, the questionnaire was used so that the students could indicate the extent to which they used metacognitive reading strategies.

The SORS comprised three subscales: Global Reading Strategies or GLOB, Problem Solving Strategies or PROB, and Support Reading Strategies or SUP. According to Mokhtari and Sheorey (2002)[17], students use Global Reading Strategies to work with text directly or to manage and monitor their reading intentionally and carefully.

Problem Solving Strategies are used for solving problems of understanding that arise during the reading of a text. Support Reading Strategies are used as basic mechanisms intended to aid reading comprehension, for example through note-taking, underlining and highlighting textual information (Mokhtari \& Sheorey 2002)[17].

SORS has demonstrated reliability and validity, the internal consistency reliability coefficient (as determined by Cronbach's alpha) was 0.92 for the Global Reading Strategies, 0.79 for the Problem Solving Strategies, and 0.87 for the Problem Solving Strategies. 
The researcher administered the questionnaires to his classes as indicated earlier, the students were asked to circle the number that applied to them indicating the frequency with which they used the reading strategy described in the statement, the students took a maximum of 20 minutes to complete the questionnaire in class under the researcher's supervision, clarification of questions were made by the researcher where and when necessary.

\subsubsection{Index of learning styles questionnaire (Isq)}

The Felder and Silverman's Index of learning styles is one of the most widely used models of learning styles, in this model, we distinguish four dimensions of learning styles, each dimension is a continuum with one learning preference on the far right and the other on the far left.

The results of the questionnaire provide an indication of individuals' learning preferences and an even better indication of the preference profile of a group of students.

The instrument consisted of 44 items that relate to learning styles, for each of the 44 questions, the student has to choose only one answer from two for each question, which seems to apply to her more frequently.

Table 1.Description oft he contentof LSQ

\begin{tabular}{|c|c|c|}
\hline Active /Reflective & 11 items & How the student prefer to process information \\
\hline Sensing/ Intuitive & 11 items & How the student prefer to take in information \\
\hline Visual/ Verbal & 11 items & How the student prefer information to be \\
& & presented \\
\hline Sequential/ Global & 11 items & How student prefer to organize information \\
\hline
\end{tabular}

\section{Data Collection}

The participants were given oral description of the objectives of the study and that their response would be used only for research purposes; participants were given 30-35 minutes to respond, and they were motivated to take the surveys seriously, and were ensured that the obtained data would be kept confidential.

\subsubsection{Data Analysis and results}

Data of metacognition survey and learning style were computed separately with the help of Excel, and then Minitab.17 was employed to compute the data gained in the study, we use the statistical procedure of data analysis for testing the relationship between metacognition and learning styles.

Our interest is to see if there is a relationship between student's metacognitive level and their learning styles.

\section{Distribution according to Learning Styles}

On the ACT/REF scale we observe that $40,5 \%$ of students are well balanced, which means they learn affectively either by doing something active with information, for example discussing, or applying it or explaining it to others, they prefer to think about information quietly, in another hand $31,6 \%$ have tendencies to action, they feel retain more information when they find ways to do something with it, $19 \%$ of students are reflective so they could retain the materials more effectively if they find ways to reflect 
Anass Hsissi, Hakim Allali, Abdelmajid Hajami; Data Analysis Application to Investigate Relationships between Metacognition and Learning Styles, Transactions on Machine Learning and Artificial Intelligence, Vol 5 No 4, August, (2017); pp: 8-19

upon them and review what they learn and think about it, finally only 3,8\% are Strongly Active, while 5,1\% are strongly reflective.

On the sensitive/Intuitive scale, students are sensing learners, $45,6 \%$ are sensitive, $10,1 \%$ are strongly sensitive, they prefer to see connections between information and real world, they don't get the whole of the material if it deals only with abstract details or theoretical data. In another hand $32,9 \%$ are well balanced, while $10,1 \%$ are intuitors , only $1,3 \%$ of students are aimed to be strongly intuitors, they have the preference to learn by discovering possibilities and relationships, in general they may have trouble with boredom in situation that deals with memorization.

We observe that $36,7 \%$ of our population have preference to visual learning style, the same percentage are strongly visual learners, they learn more effectively with any type of visual representation of course material, while only $5,1 \%$, and $1,3 \%$ aimed to be respectively verbal and strongly verbal, they get more out of words, written and spoken explanations.

On the scale of sequential and global learning style, we have observed that $36,7 \%$ are well balanced in that scale, $13,9 \%$ are aimed to prefer Global learning style, while $1,3 \%$ of the population is very global, they tend to learn in large jumps, learn almost randomly without seeing connections. $38 \%$ of students prefer the sequential style, they tend to learn in linear steps, the more they put the material in a logical path the more they feel comfortable and the best they learn.

We confirm that everybody is for example, active sometimes and reflective sometimes, the same for the other scale, but the strong or the moderated preference for one or the other which highlights some cautions, if the student overemphasize intuition he may miss important details or make some mistakes, if he overemphasize sensing he may rely too much on memorization and not concentrate enough on critical and innovative thinking.

\section{Principal component analysis}

In order to get knowledge of our experimental data, we choose to perform dimensionality reduction of the space, while preserving as much of the randomness in the high dimensional space as possible [18], it is a basic idea behind Principal component analysis, here we take the cloud of observations or points, and rotates to make visible the maximum variability.

PCA is a data analysis technique to identify relations between features; we use it to extract and select features and to find response related to our research guiding questions.

The task of investigation is a feature extraction and selection method, the goal is to obtain the most relevant information from the observation and features regardinglearning styles and metacognitive awareness and represent that information in a lower dimensionality space, our questions concerns the relations between the way students prefer to learn, as learning styles, and the awareness in learning as metacognition, to discover important features of data set, and interpret to construct a useful analysis.

We conduct our analysis on a multidimensional space of data representing the profiles of learners and their metacognitive skills, we will highlight in the next part of the paper the relationships between styles in learning and Metacognitive problem solving skills, then in the discussion we will provide you with further details. 


\subsubsection{Learning styles and Metacognitive Problem Solving Strategies.}

The eigenvalues indicate that three components provide a reasonable summary of the original information, accounting for $70 \%$ of the total variance.

The table of principal components (Table 5) reveals a strong correlation between Problem solving strategies variable and being sequential, active and sensitive,( SN 0.414 SEQ 0.266) on the principal component one, but this kind of skills is affected positively with reflection (RF -0.583 M_MetaPRO -0.493).

Table 2.Tableofprincipalcomponentsanalysis

\begin{tabular}{|cccc|}
\hline Variable & PC1 & PC2 & PC3 \\
\hline AC & 0,202 & 0,583 & $-0,058$ \\
\hline RF & $-0,181$ & $-0,608$ & 0,072 \\
\hline SN & 0,414 & 0,143 & 0,058 \\
\hline IN & $-0,414$ & $-0,139$ & $-0,053$ \\
\hline VI & $-0,018$ & 0,046 & $-0,624$ \\
\hline VR & 0,014 & $-0,047$ & 0,623 \\
\hline SEQ & 0,266 & $-0,039$ & 0,267 \\
\hline GL & $-0,303$ & 0,041 & $-0,238$ \\
\hline M_MetaPRO & 0,649 & $-0,493$ & $-0,284$ \\
\hline
\end{tabular}

The first component will follow that students with large sense of sensitivity in learning, and also logical information processing, whereas communities with small values would have very few of these types of characteristics (Fig.6).

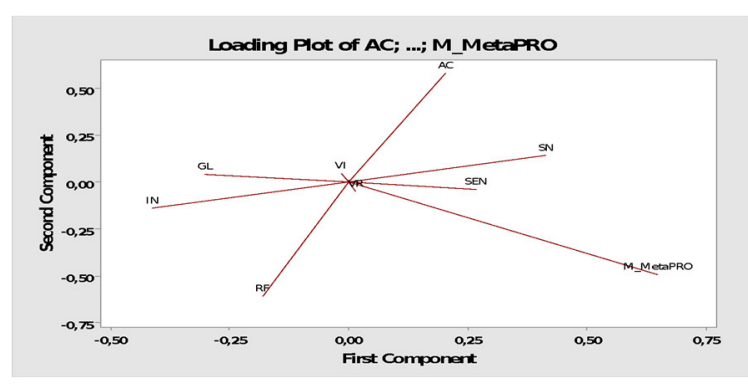

Figure 1. Loading plot of learning styles and Problem solving strategy

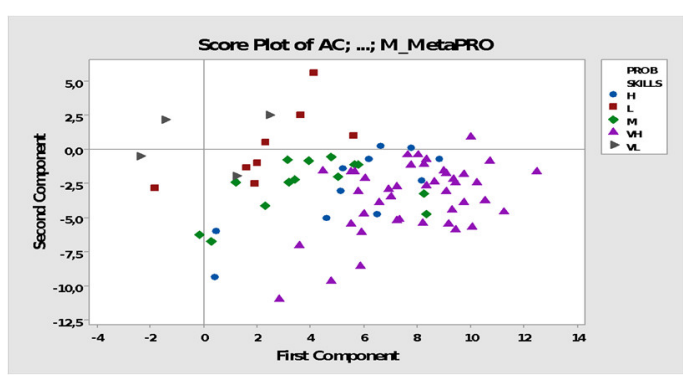

Figure 2. Score plot in dicating level of mastery of Problem solving strategy

To complete the analysis of learning styles and problem solving skills, we produce a scatter plot of the component scores, where we plot the second against the first component, (see Figure.1) each dot represent one student, we give them five kind of symbols by degree of proficiency in problem solving skills, in this present context, we wish to identify the location of the points to see if students with high levels of given component tend to be clustered in a particular manner or a particular region of the plot. Effectively student with high proficiency are situated in the region with high value for the first component $(\mathrm{VH}, \mathrm{H}, \mathrm{M})$, the student with grade VL-Very Low have a low values, these poor student are more active, less sequential and less sensitive than others. 


\section{Discussion}

In an effort to investigate the relationships between the way learners control and monitor learning process (metacognition), and their preferences as learning styles, we performed this case study, we apply data analysis to explore relations and do useful interpretations using principal component analysis, we overcome the multidimensionality of the dataset, we identify the relations and patterns behind the data of the experimentation.

As conclusion, metacognition is strongly related to learning styles, this is probably due to the importance of the personal identity of learners, each one learn according to some criteria, which may hurts learning in some cases when environment don't enhance those preferences.

\subsection{Learning styles affects global thinking strategies}

Global Reading strategies (GLOB), which can be thought of as generalized strategies, it aim is setting the stage for reading act, we consider this element as the basic strategies of reading for comprehension, localized at the start point of the learning act, focused on evaluating what to read, what to ignore, setting goal, noting characteristics of the task, guessing, etc.. All this strategies are more or less affected by styles of learning.

The first finding of this research reinforce the idea that Intuition and Global learning preferences affects positively the way every learner control and monitor his learning process; the Visual and the balance between Active and Reflective preferences might help to enhance metacognition, while Sequential style may hurts global strategies. The first component will follow that students who are global, with large sense of intuition in learning, whereas communities with small values would have very few of these types of characteristics, the second component will follow active and visual preferences. As shown in Fig.14,both the second component (Y1) as described in the first finding and the first (X1) affects the level of global strategy; the darker green regions indicate higher quality.

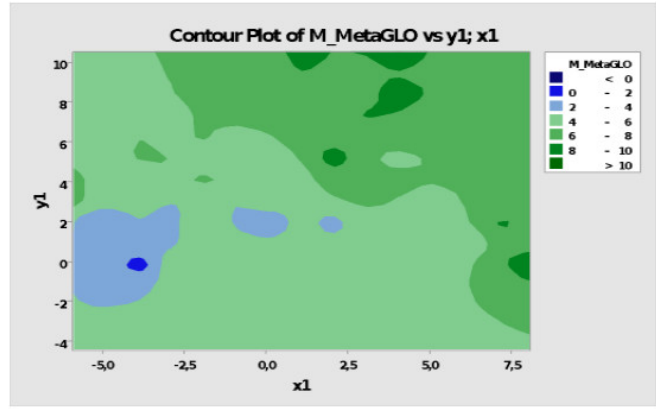

Figure.3: Contour plot describing level obtained on global strategy on a numeric scale

From the definition of intuition, which can be developed with accumulating experience, after working on several situations, our results confirm the importance of this element on making reasonable connections without formalizing the arguments. On other hand global preference came to adjust intuition, her place is not to destroy all intuition, so according to our results on global metacognitive skills, we claim that global thinking should be used to destroy bad outcomes of intuition, while clarifying and elevating good intuitions. We propose that only good combinations of both global processing and intuition that can lead 
learner to tackle learning problems, the first (Global style) helps to deal with the big picture while the latter (Intuitive style) helps to deal with the fine details.

\subsubsection{Learning styles are the keys to enhance problem solving strategies}

Problem solving strategies are used when problems arises, known as repair strategies they enable learners to overcome trouble, control understanding, for example in case of losing concentration, learner may decide to go back for better understanding.

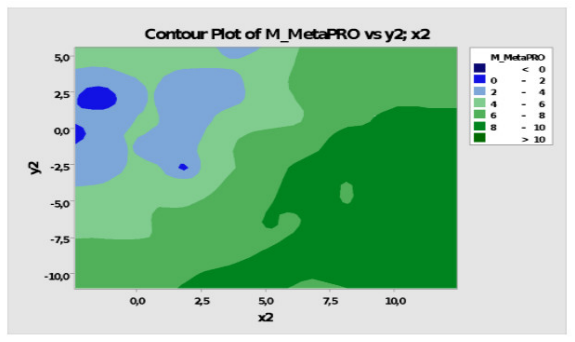

Figure. 1:Contour plot describing level obtained on problem solving strategy on a numeric scale

For this element we confirm that z Reflective, Sequential and Sensitive learning styles are the best styles to boost problem-solving strategies.

The first component (X2) will follow that students with large sense of sensitivity in learning, and also logical information processing, whereas communities with small values would have very few of these types of characteristics, the following contour plot (Fig.15) explores the potential relationship between the two components and the problem solving skills.

We have seen that problem solving strategy is related to both sensitivity and reflection as learning styles, this results ally with findings related to this type of strategy in learning, since sensing is considered as the ability to learn by manipulating objects, skills about learning inductively rather than deductively. In general sensitive learners tend toward psychomotor over abstract thinking, they prefer personal connections to topics, they follow directions they have written themselves, and they benefit more from demonstrations. The second element is reflection, enables learners to reflect upon their results and outcomes, in order to adjust and monitor eventual future actions, we suggest that getting best performance in problem solving strategy is enhanced by fostering sensing and reflection in learning.

\subsection{Support strategies are influenced by learning styles}

Support strategies are the third broad subcategory measured, it consists of providing learners with additional support mechanism, the third finding of this research confirms the idea that SENSITIVE, ACTIVE, VERBAL learning preferences following the first component (X3), has all positive influence on the way learners search for additional support, using Support strategies.

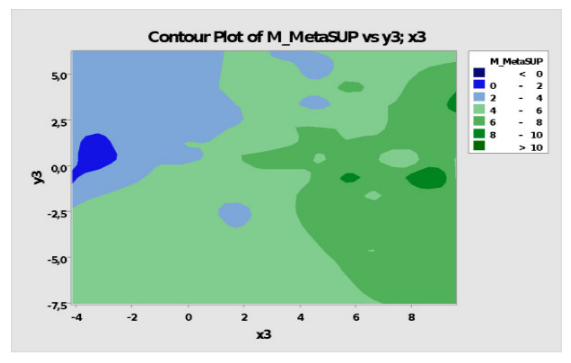


Anass Hsissi, Hakim Allali, Abdelmajid Hajami; Data Analysis Application to Investigate Relationships between Metacognition and Learning Styles, Transactions on Machine Learning and Artificial Intelligence, Vol 5 No 4, August, (2017); pp: 8-19

Figure. 2: Contour plot describing level obtained on support strategy on a numeric scale

Support strategy can be enhanced by fostering sensitivity in learning, active processing and verbal instructions, effectively learner benefits from those preferences to enhance their level of proficiency, support reading strategies are used as basic mechanisms intended to aid reading comprehension such as note-taking, underlining and highlighting textual information, those qualities might be enhanced with some aids such as collaboration, concept map, and feedbacks.

\section{Conclusion}

In the previous part we present a summary of our findings, and explanation of how it confirms what was described in the literature, the present study suggests that understanding learning styles of learners might contribute to increase the efficiency and quality of learning, good identification of each one's preferences, weaknesses and strengths is the key to make effective learning happen, we have used principal component analysis as a tool to apply an exploratory data analysis and predictive analysis, to explain the variation in data, it allows us to see how different variable change with each other, and enable us to select a minimum number of principal components to explain most of the variation and to select which variables are the most significant in describing the full data set. This study may provide educational with good insights about cognitive process involved in learning, and what differentiates successful from less successful students. Because understanding begins to develop when students have frequent opportunities to deploy some strategies to control and monitor the reading process, this ability can be enhanced as they have more opportunities, more support, and encouragement.

Actually we work on several implications for instructional interventions in e-learning context, our focus is to teach students how to be more aware of their learning processes and products, as well as how to monitor and regulate those processes for an effective learning. We believe that building intelligent environment for human learning, with additional care for styles of learning, motivation and individualization can permit advances in the area of metacognition and e-learning.

\section{REFERENCES}

[1] J. H. Flavell, 1979. Metacognition and cognitive monitoring: A new area of cognitive developmental inquiry. American Psychologist, 34(10), 906-911.

[2] Nelson \& Narens, 1994. Why investigate metacognition ? In J.Metcalfe \& AP. Shimamura (Eds). Metacognition: knowing about knowing (pp. 1-26). Cambridge, MA: MIT Press

[3] Schraw \& Dennison, 1994. Assessing metacognition awareness, Contemporary Educational Psychology,19, 460-475

[4] Pintrich, \& Zeidner, 2000. The role of goal orientation in self-regulated learning. Handbook of self-regulation (pp, 452-494), San Diego, CA: Academic Press

[5] Harris, T.L, \& Hodges, R.E, 1995. The literacy dictionary: The vocabulary of reading and writing. Newark, DE: internationai Reading Association 
Transactions on Machine Learning and Artificial Intelligence Vol 5 No 4, Aug 2017

[6] Livingstone .J.A, 1996. Effects of metacognitive instruction on strategy use of college students. Unpublished manuscript, State University of New York at Buffalo.

[7] Caliskan, M., \& Sunbul, A, 2011. The effects of learning strategies instruction on meta-cognitive knowledge, using meta-cognitive skills and academic achievement, primary education sixth grade Turkish course sample. Educational Sciences: Theory \& Practice, 11(1), 148-153

[8] Hoskin, P. W. O, 2000. Affecting increased student achievement in geoscience education by instruction in metacognition: A small class case study. Retrieved from http://www.usq.edu. au/electpub/ejist/docs/Vol7_no2/CurrentPractice/Affect_student_ach.htm

[9] Baumann \& Duffy, 1997 Engaged Reading for Pleasure and Learning: A Report from the National Reading Research Center.

[10] Downing, K., Kwong, T., Chan, S., Lam, T. and Downing, W, 2009. Problem-based learning and the development of metacognition. Higher Education, 57 (5), 609-621.

[11] Singhal, 2001. Reading proficiency, reading strategies, metacognitive awareness and L2 readers. Reading Matrix, 1-9

[12] Oxford, R. L, 1990. Language learning strategies: What every teacher should know. USA: Heinle \& Heinle

[13] Bianco, M, 2010. La compréhension des textes : peut-on l'apprendre et l'enseigner ? In M. Crahay \& M. Dutrevis (Eds.), Psychologie des apprentissages scolaires, (pp. 229-256). Bruxelles: De Boeck.

[14] Nelson, S., \& Conner, C, 2008. Developing self-directed learners. Retrieved January 15, 2008

[15] R. Felder and J.Spurlin. "Applications, Reliability, and Validity of the Index of Learning Styles,"

[16] Efklides, 2006. Metacognition and affect: What can metacognitive experiences tell us about the learning process?. Educational Research Review, 1, 3-14

[17] Mokhtari and Sheorey, 2001. Differences in the metacognitive awareness of reading strategies among native and non-native readers. System, 29, 431-449.

[18] I.T.Jolliffe. Principal component analysis, Second Edition, Springer, 2nd ed. P.cm - Springer series in statistics, ISBN 0-387-95442-2

[19] Jackson, J.E . 1991, A user's guide to Principal Components. New York : Wiley. 